Highly Energetic Physical Processes and

Mechanisms for Emission from Astrophysical Plasmas

IAU Symposium, Vol. 195, 2000

P. C. H. Martens, S. Tsuruta, and M. A. Weber, eds.

\title{
Interaction of the SS433 Jet with the Interstellar Medium
}

\author{
P. O'Neill and R. Sood
}

School of Physics, University College, University of New South Wales, Australian Defence Force Academy, Canberra, ACT 2600, Australia

P. Durouchoux

CE Saclay, DSM, DAPNIA, Service d'Astrophysique, Cedex, France

\author{
S. Safi-Harb \\ NASA, GSFC, Code 662, Greenbelt, MD 20771, U.S.A.
}

\begin{abstract}
X-ray emission observed from the SS433/W50 system has been interpreted as resulting from the interaction of the binary jets with the surrounding medium. We have been carrying out millimetre wavelength measurements of this system. We find no evidence for the association of a molecular cloud with the eastern jet, but we do find evidence for a strong association with the western jet.
\end{abstract}

\section{Introduction}

SS433 is an X-ray binary located at the centre of the supernova remnant W50 (Margon 1984; Safi-Harb \& Ogelman 1997). Two jets are associated with the object, which move in opposite directions at $0.26 \mathrm{c}$. The jets precess with a period of 163 days. Figure 1a shows ROSAT X-ray contours of W50 (Safi-Harb \& Ogelman 1997).

To determine the level of interaction between the jets and an associated molecular cloud, we have established a millimetre wavelength observing programme involving measurements of $\mathrm{CO}, \mathrm{SiO}$, and $\mathrm{HCO}^{+}$line transitions. Here, we review our previous observations (Durouchoux et al. 2000) and present recent results.

\section{Observations}

Previous observations were carried out using the Nobeyama, SEST, and Mopra telescopes in Jan., Mar., Apr., May and Oct. 1998. We detected no CO emission in the eastern jet $\left(e_{1}, e_{2}\right.$ and $e_{3}$; see Figure 1a). Measurements of the western jet region, marked in Figure 1a as "MOPRA" and 10, revealed a correlation between ${ }^{12} \mathrm{CO}(1-0)$ emission at $50 \mathrm{~km} \mathrm{~s}^{-1}$ and the X-ray contours (Durouchoux et al. 2000).

During 16-21 Apr. 1999, we measured the ${ }^{12} \mathrm{CO}(1-0)$ emission at $50 \mathrm{~km} \mathrm{~s}^{-1}$ in the regions marked 10 to 13 in Figure 1a. The resulting map is shown in 
Figure 1b. There is little signal from the regions outside the jet cone, while there is a strong signal from within the cone.
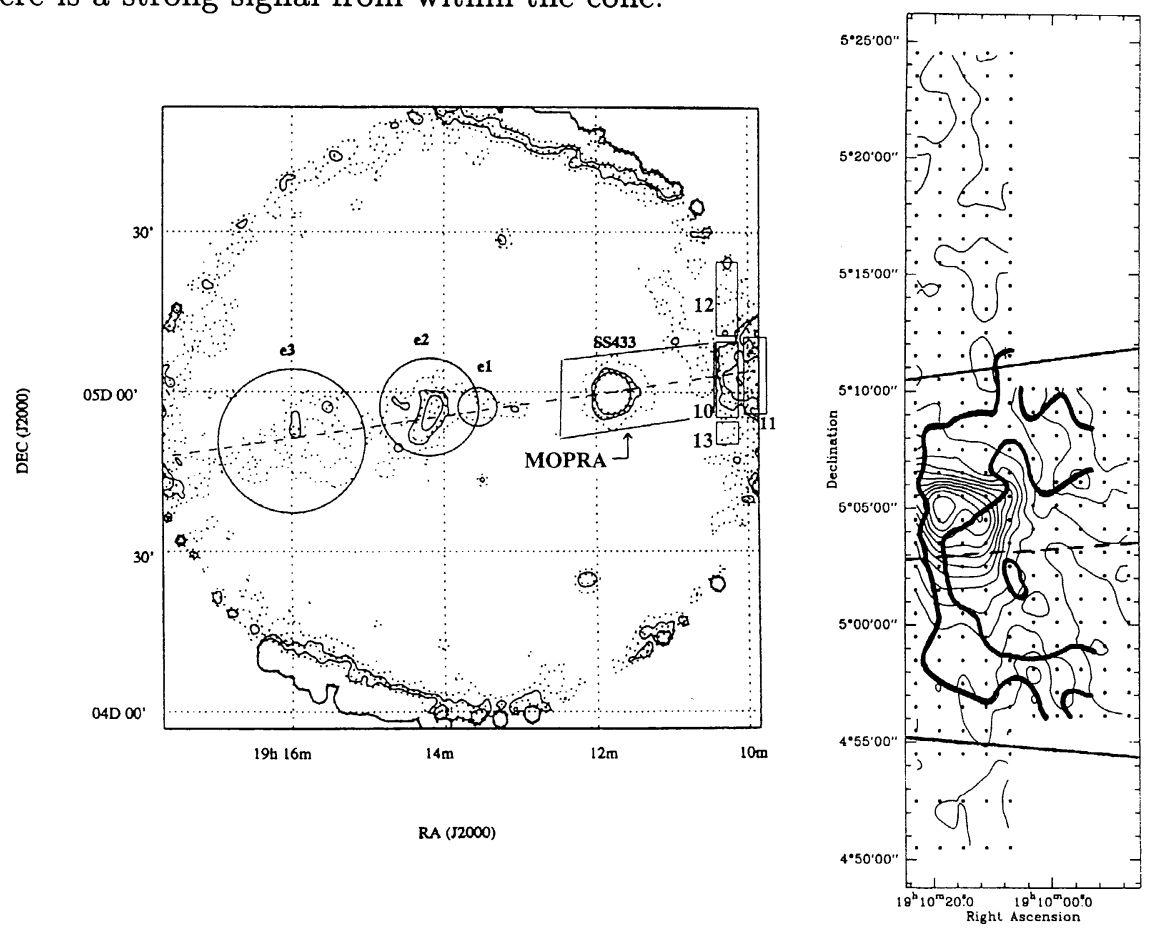

Figure 1. (a, left) X-ray emission associated with SS433/W50. The dashed line is the jet precession axis. (b, right) ${ }^{12} \mathrm{CO}(1-0)$ emission map. The bold solid lines are the X-ray intensity contours. The thinner solid lines show the precession cone.

The data suggest there is a correlation between the $50 \mathrm{~km} \mathrm{~s}^{-1}$ molecular cloud and SS433. Figure 1b shows evidence for a shock front, located to the north of the precession axis, which we propose is generated by the interaction of the jet with the molecular cloud. Limited observations of ${ }^{13} \mathrm{CO}(1-0)$, an indicator of column density, provide further evidence for the interaction.

Future observations will attempt to trace the expected spatial shift of the shock front with the precession of the jet.

\section{References}

Durouchoux, P., et al. 2000, Adv. Space Res., in press

Margon, B. 1984, ARA\&A, 22, 507

Safi-Harb, S., \& Ogelman, H. 1997, ApJ, 483, 868 